\section{$\underset{\substack{\text { hommes } \\ \text { \& migrations }}}{ }$}

\section{Hommes \& migrations}

Revue française de référence sur les dynamiques

migratoires

1285 | 2010

L'appel du pied

\title{
Saïd Bouziri et Mohamed "Mokhtar" Bachiri
}

Deux figures de l'immigration aux parcours contrasté

\section{Mogniss H. Abdallah}

\section{Q OpenEdition}

\section{Journals}

\section{Édition électronique}

URL : http://journals.openedition.org/hommesmigrations/1203

DOI : 10.4000/hommesmigrations. 1203

ISSN : 2262-3353

\section{Éditeur}

Musée national de l'histoire de l'immigration

\section{Édition imprimée}

Date de publication : 1 mai 2010

Pagination : 172-176

ISSN : 1142-852X

\section{Référence électronique}

Mogniss H. Abdallah, "Saïd Bouziri et Mohamed "Mokhtar" Bachiri », Hommes \& migrations [En ligne],

1285 | 2010, mis en ligne le 29 mai 2013, consulté le 22 septembre 2020. URL : http://

journals.openedition.org/hommesmigrations/1203; DOI : https://doi.org/10.4000/

hommesmigrations. 1203

Ce document a été généré automatiquement le 22 septembre 2020.

Tous droits réservés 


\title{
Saïd Bouziri et Mohamed "Mokhtar" Bachiri
}

\author{
Deux figures de l'immigration aux parcours contrasté
}

\author{
Mogniss H. Abdallah
}

1 La disparition soudaine de Saïd Bouziri, le 23 juin 2009, a abasourdi ceux qui l'ont connu. Figure incontournable de l'histoire de l'immigration, il était aussi un personnage respecté du milieu associatif, tant au niveau local à Barbès que sur le plan national. Nombreux ont donc été les gens venus lui rendre hommage salle Saint-Bruno, là même où, trente-sept ans auparavant, il avait défrayé la chronique en menant avec sa femme Fawzia une grève de la faim contre une mesure d'expulsion du territoire prise à leur encontre.

2 À la levée du corps, parmi les amis, compagnons de route, personnalités politiques ou représentants de la société civile, on a remarqué la présence de Mohamed Bachiri, dit "Mokhtar". Beaucoup avaient perdu de vue ce tribun tumultueux qui forma avec Saïd un tandem contrasté lors des grandes luttes du Mouvement des travailleurs arabes (MTA) dans les années soixante-dix. L'un tissait des réseaux tout en montant les dossiers juridiques et en gérant l'intendance, l'autre galvanisait les foules par sa parole crue lors des A. G., puis au micro de Radio Soleil Goutte d'Or. Mais courant des années quatre-vingt, l'un et l'autre ont emprunté des voies différentes et, depuis la brusque fin de "sa" radio en 1987, Mokhtar était retourné travailler à l'usine comme tôlier intérimaire en France ou ailleurs en Europe.

Depuis, il était devenu injoignable. Les ponts semblaient définitivement rompus. Et son mutisme laissa planer comme un angoissant non-dit collectif. Aussi, de par sa seule présence pour saluer une dernière fois Saïd, il a provoqué parmi l'assistance un soulagement réparateur, signifiant par là même qu'au-delà des conflits passés, les uns et les autres pouvaient se reparler dans le respect mutuel. À cette occasion, il a pu reprendre langue avec son compatriote Driss El Yazami, un des plus proches collaborateurs de Saïd Bouziri au sein de l'association Génériques. Mais Mokhtar était malade, et c'est avec une grande tristesse que ses amis retrouvés ont appris sa disparition le 3 février 2010. 
4 À travers l'émotion suscitée par l'évocation de Saïd Bouziri et Mokhtar Mohamed Bachiri, tout un pan de l'histoire commune remonte à la surface, fait de parcours singuliers et d'expériences croisées.

\section{Le coordinateur et le tribun}

5 Né à Tunis en 1947, Saïd Bouziri est l'aîné des enfants d'une famille de commerçants réputés. Il arrive en France en 1966 et poursuit à Lyon des études d'économie, avant de s'installer à Paris où il exercera toute sa vie professionnelle comme comptable, pour l'essentiel à l'Unédic. Après la guerre israélo-arabe de juin 1967 et Mai 68, il s'engage pour la cause palestinienne et s'intéresse à la condition sociale des travailleurs immigrés. Il côtoie les cercles universitaires marxistes-léninistes arabes et français, se rapprochera de la "Gauche prolétarienne" (GP) sans forcément en partager toutes les idées ou pratiques. Il s'attache ainsi davantage à la situation de non-droit des immigrés dans les quartiers et au racisme qui en découle. Saïd Bouziri et son frère Hamza iront souvent, à la porte des usines, au contact des ouvriers parfois méfiants vis-à-vis des militants extérieurs. C'était le cas de Mohamed Bachiri qui, déjà débordé par les conditions de travail à Chausson Gennevilliers ou à Renault Flins et par les premières bagarres dans les foyers Sonacotra, se montra d'abord réticent face aux sollicitations des frères Bouziri, qui pressentaient en lui "le plus grand tribun chez nous qui déplaçait les foules"1. Ils devront user de beaucoup de persuasion pour le convaincre de devenir le porte-parole des Comités Palestine puis du MTA.

6 Mokhtar, né en 1947 à Sidi Boubker près d'Oujda (Maroc), est l'aîné dans une famille dont le père militant nationaliste et syndicaliste travaillait dans les mines de Zellidja, découvertes par l'architecte alsacien Jean Welter. Mokhtar se fera virer de la mine pour avoir rossé un policier. Jeune et rebelle au chômage, il ne quitte alors plus le poste de radio de son père. Sa formation politique, il la fera d'abord à l'écoute de la Voix des Arabes du Caire, et des radios qui couvrent Mai 68 en France. Son assiduité radiophonique et la culture orale traditionnelle qui lui est transmise développent ses capacités de mémorisation et son sens de la formule qui galvanise. Puis il est recruté à Casablanca en 1969 pour aller bosser à Chausson où "ça commence à travailler dans la tête ; je vais à l'usine, le travail est dur, les chefs gueulent, les cadences me rendent fou, la paye est mauvaise. Je reviens chez moi, le gérant gueule, j'ai pas le temps de faire la cuisine... Alors à peine le contrat fini - un contrat de six mois - on quitte tous Chausson. On dit qu'on va aller autre part." Au contact des maos, il se dit que "pour résister... il fallait s'organiser"

7 Entre 1971 et 1973, les mobilisations immigrées vont se bousculer : meurtre raciste du jeune Djillali Ben Ali, quadrillage policier de Barbès, entrée en vigueur des circulaires Marcellin-Fontanet, mesure d'expulsion à l'encontre de Saïd et Fawzia Bouziri, mort de Mohamed Diab au commissariat de Versailles, grèves de la faim des sans-papiers pour la carte de travail et la régularisation... Sur tous ces sujets, Saïd Bouziri et Mokhtar se révéleront de redoutables agitateurs. Il s'agissait aussi de "bombarder l'opinion publique et les moyens d'information"'. Or, selon l'administration, les étrangers sont alors tenus à la neutralité politique, et c'est pour avoir enfreint cette règle que les Bouziri ont été menacés d'expulsion. Dans le même temps, des divergences de vues sur la question palestinienne renforcent l'idée de la nécessaire autonomie du MTA naissant, tant sur le plan de l'organisation politique que de l'action culturelle. Pas question pour autant de repli communautaire, ni de distendre les liens étroits tissés avec les milieux chrétiens 
ou syndicaux et les intellectuels. Ces derniers, parmi lesquels Claude Mauriac, Jean-Paul Sartre et Michel Foucault, participent au Comité de défense de la vie et des droits des travailleurs immigrés (CDVDTI ), constitué dans le prolongement de la campagne "victorieuse" pour les Bouziri. Saïd contribue ainsi au lancement du quotidien Libération, s'investit pour les droits des chômeurs et fréquente des leaders médiatiques qui deviendront plus tard des décideurs, de gauche ou de droite, arpentant les allées du pouvoir.

Mokhtar fonde pour sa part avec Geneviève Clancy et Philippe Tancelin du CDVDTI la troupe Al Assifa, qui présentera pour la première fois son "journal théâtral" intitulé "Ça travaille, ça travaille et ça ferme sa gueule" dans l'usine Lip occupée (été 1973). Cette troupe, qui se produira aussi au festival d'Avignon, connaitra de multiples déclinaisons, y compris une version en cassette audio, "Radio Assifa", coréalisée en 1975 avec Hamza Bouziri. Elle participera à différentes éditions du Festival du théâtre populaire des travailleurs immigrés entre 1975 et 1982. Enfin, Mokhtar donnera volontiers un coup de main à La Kahina, Week-end à Nanterre ou Mohamed Travolta, des groupes montés par les nouvelles générations héritières de l'immigration ${ }^{4}$.

\section{Crise du militantisme et enjeux mémoriels}

9 Cependant, une crise récurrente couve au MTA. Saïd Bouziri critique "l'opportunisme militant" et "l'esprit de bande" qui vit en "vase clos"5. Plus tard, il reconnaîtra avoir beaucoup fonctionné sur le mode de "l'évitement", pour ne pas engendrer une organisation autoritaire et ne pas alimenter les zizanies interpersonnelles. En même temps, "avec le recul, je peux dire qu'on n'avait aucune idée de nos objectifs"'.

Il se recentre sur Barbès, autour de l'Association socioculturelle de la Goutte d'Or, siège du journal Sans Frontière en 1979, et d'où émettra Radio Soleil en 1981. Mokhtar, virtuose au micro, anime l'antenne et gagne un vaste auditoire immigré et populaire aux ressources insoupçonnées. Mais il se montre réservé vis-à-vis du journal et son équipe composée en partie d'anciens du MTA. D'après lui, Sans Frontière dégénère en instrument de négociation avec les pouvoirs publics et se compromet avec la nouvelle politique officielle de "la fin des immigrés". Ressurgit alors en Mokhtar son aversion épidermique pour la politique politicienne. Les relations s'enveniment au point de voir deux Radios Soleil concurrentes, l'une à la Goutte d'or, l'autre à Ménilmontant. Saïd Bouziri estime lui, qu'avec le droit d'association et d'expression désormais acquis, il faut continuer le "grignotage" pour obtenir plus. Fin négociateur ayant le souci du long terme, il intègre les comités d'administration du FAS et de la Fonda, fort de l'idée qu'il faut désormais investir les institutions et les associations généralistes afin d'établir un partenariat actif avec elles. Il se méfie en effet de la logique du "deuxième collège" qui confierait aux associations un rôle subalterne ou supplétif. Aussi se hisse-t-il à la direction de la Ligue des droits de l'homme, où il tente de mener de pair luttes locales ou spécifiques et luttes générales. Jusqu'à la fin de sa vie, il impulsera ainsi au nom de la LDH la campagne "Votation citoyenne" en faveur du droit de vote des résidents étrangers, et le collectif national Associations en danger. Néanmoins, malgré un parti pris institutionnel de plus en plus affirmé, il gardera une réelle affection pour l'expression spontanée du militantisme d'antan, nourrissant le secret espoir qu'elle réémerge sous des formes renouvelées. Comment expliquer autrement sa participation 
discrète mais assidue à une kyrielle de petites associations et sa présence aux côtés des nouveaux sans-papiers chinois ou autres?

11 Mokhtar, lui, malgré ses désillusions et son retrait de la scène publique, semblait presque apaisé suite à ses voyages sans encombre au Maroc à partir de 1986. Selon ses proches, il avait aussi renoué avec la fraternité ouvrière auprès de ses amis et collègues de travail. Enfin, à Zellidja, il aidait ses copains d'enfance à monter des micro-projets et partageait son savoir-faire original en tôlerie. À sa manière, il poursuivait ainsi, autrement, le combat du MTA pour redonner de l'importance aux gens ordinaires, dont l'histoire mérite d'être racontée au même titre que celle des militants. Malheureusement, au terme d'une vie tourmentée, ses archives ont été dispersées ${ }^{8}$. Par anticipation, Saïd Bouziri avait, dès 1990, déposé une partie de ses archives propres à la BDIC de Nanterre. Et, avec Génériques, il a encouragé les autres à faire de même, en soulignant bien que les dépôts publics n'entraînaient pas une dépossession mémorielle. À condition sans doute que la réappropriation des histoires de vie par les acteurs euxmêmes et leur inscription dans la mémoire collective soient partagées comme un enjeu majeur. Les parcours de Saïd Bouziri et de Mokhtar Mohamed Bachiri invitent à ce partage, et nous incitent à la poursuite d'une réflexion encore parcellaire et inachevée sur l'évolution de l'engagement personnel dans des contextes en constante mutation.

\section{NOTES}

1. Saïd Bouziri, in Migrance $\mathrm{n}^{\circ} 25,1^{\mathrm{er}}$ trimestre 2005.

2. Cf. entretien avec Michèle Manceaux in Les Maosen France, Paris, Gallimard, 1972.

3. In APL informations $n^{\circ}$ 59, 8 octobre 1972. Voir aussi Jean Benoît, E comme Esclaves, Paris, éd. Alain Moreau, 1980.

4. Sur l'histoire d'Al Assifa, voir Geneviève Clancy et Philippe Tancelin in Les Tiers idées, Paris, éd. Hachette 1976.

5. Crise ouverte dans le MTA à Paris, juillet 1976, document ronéotypé.

6. Cf. Migrance, op. cit. ; et Saïd Bouziri par lui-même : www.dailymotion.com/Agence_Im-media.

7. Le dernier numéro collector de Sans Frontière, en avril 1984, titre "Tchao l'immigration!?”

8. Un appel à contribution pour le recueil d'archives (photos, écrits, films...) a été lancé le 13 mars 2010 à Paris. L'association Les Enfants de Zellidja a mis en ligne les premiers documents retrouvés : www.zellidjaboubeker.net/forum.html. 